\title{
Multicolor Single Molecule Tracking of Stochastically Active Synthetic Dyes
}

\author{
Alexander Benke, ${ }^{\ddagger}$ Nicolas Olivier, ${ }^{\ddagger}$ Julia Gunzenhäuser, and Suliana Manley* \\ Laboratory of Experimental Biophysics, EPFL, 1015 Lausanne, Switzerland
}

Supporting Information

\begin{abstract}
Single particle tracking can reveal dynamic information at the scale of single molecules in living cells but thus far has been limited either in the range of potential protein targets or in the quality and number of tracks attainable. We demonstrate a new approach to single molecule tracking by using the blinking properties of synthetic dyes targeted to proteins of interest with genetically encoded tags to generate high-density tracks while maintaining flexibility in protein labeling. We track membrane proteins using different combinations of dyes and show that the concept can be extended to three-color imaging. Moreover, we show that this technique is not limited to the membrane by performing live tracking of proteins in intracellular compartments.
\end{abstract}
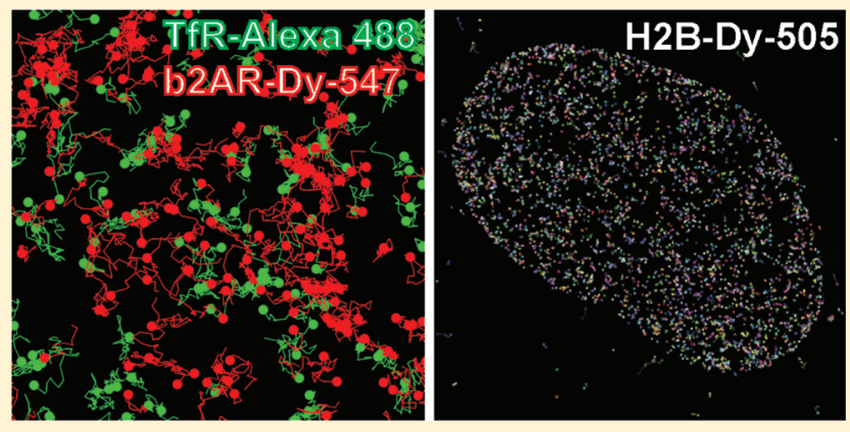

KEYWORDS: single molecule tracking, multicolor tracking, protein dynamics, super-resolution imaging

$\mathrm{D}$ ynamic measurements of single molecules in living cells offer a window into nanoscale processes obscured by conventional imaging methods. Single molecule tracking in living cells has been improved by exploiting temporal separation of signals to allow up to thousands of molecules to be tracked in a single cell in the time span of minutes. ${ }^{1}$ This has been implemented using two different approaches to fluorescently label proteins of interest either via protein fusions to photoswitchable or photoactivatable fluorescent proteins ${ }^{1}$ or more recently via ligand binding. ${ }^{2}$ In the case of protein fusions, there are few constraints on the nature of the protein of interest; since dyes are genetically encoded proteins, they can be studied in virtually any cellular compartment. However, the relatively low photon yields of fluorescent proteins limit the time scales over which a given molecule can be tracked, and the accuracy with which it can be localized. In the case of ligand binding, ligands are conjugated to synthetic dyes that have high photostability and long lifetimes but limit the possible proteins studied to extracellular receptors with efficiently targeted ligands. Moreover, the pool of unactivated receptors without a ligand bound is invisible to this method. Here, we combine the advantages of protein target flexibility and dye properties by using genetically encoded tags to target synthetic dyes as a means of probing the dynamics of membrane and intracellular proteins.

Genetically encoded protein tags enable rapid and specific protein labeling via the formation of a stable, covalent bond between the protein and the label. ${ }^{3}$ Thus, they offer an interesting alternative to other forms of labeling by fluorescent proteins or ligands. Unlike antibody labeling, they are also compatible with live-cell imaging. Self-labeling proteins, such as the SNAP-tag ${ }^{4}$ and Halo-tag, ${ }^{5}$ which are smaller than fluorescent proteins, are fused to the protein of interest and react directly with the labeling compound, benzylguanine (BG) or Halo. These two tags have been used in live-cell imaging, including super-resolution imaging. ${ }^{6-9}$ Enzyme-mediated tags are even smaller than self-labeling proteins and have higher specificity but require the addition of an enzyme to covalently link the label to the tag and therefore can only be used extracellularly. ${ }^{3}$ We combine both self-labeling proteins and enzyme-mediated tags for targeting synthetic dyes, which we then use to track single molecules with multiple colors and in different cellular compartments. To track thousands of molecules in a single cell, we take advantage of the photophysical processes that allow fluorophores to cycle through reversible dark states. These processes allow us to control the number of molecules that are in a fluorescent state at any given time. This is the same mechanism for temporal separation of fluorescent signals exploited to create superresolution images. ${ }^{10-12}$

Temporal separation of single molecule signals can be obtained by a number of different mechanisms, including chemical control of the rate of recovery from a dark state. ${ }^{13} \mathrm{~A}$ combination of $\beta$-mercaptoethanol (BME) and oxygen scavengers was originally used to control the dark states of Cy5 dyes and enable point localization of single molecules. ${ }^{11}$ Since then, other thiols, such as cysteamine (MEA), have been used in combination with oxygen scavengers to induce the

Received: March 15, 2012

Revised: April 17, 2012 

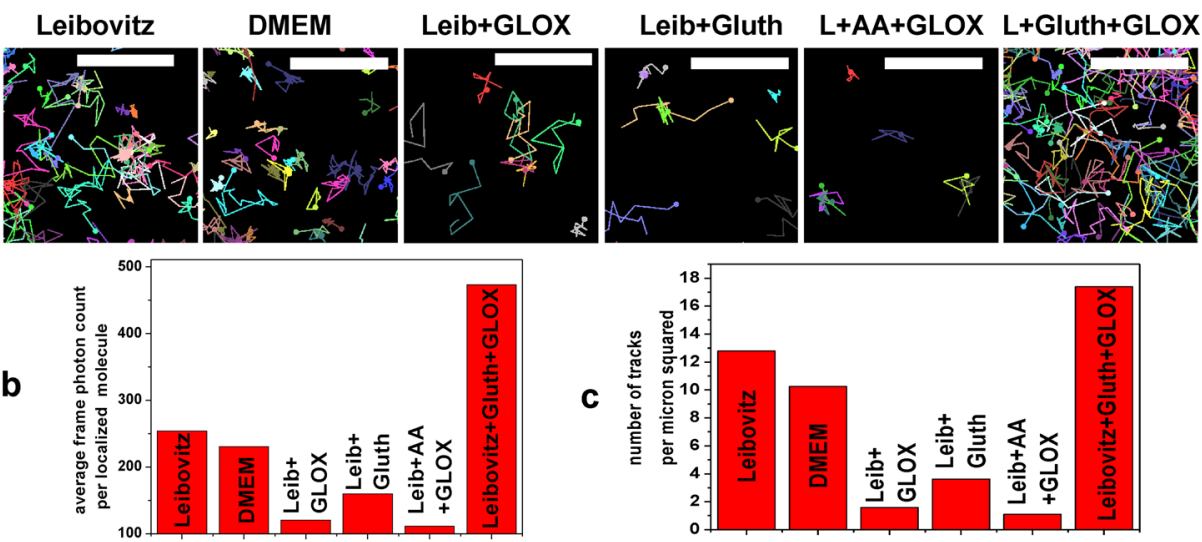

Figure 1. (a) Example of typical SNAP- $\beta 2 A R$ tracks obtained in the different buffer conditions tested on a $2 \times 2 \mu \mathrm{m}$ area (scale bar $=1 \mu \mathrm{m})$. The starting point of each track is indicated by a dot. (b) Average number of detected photon per track point (threshold set at 100 photons) and (c) average track density per micrometer squared (minimum track size: 8 points) measured in 5000 frames for the different buffer conditions.

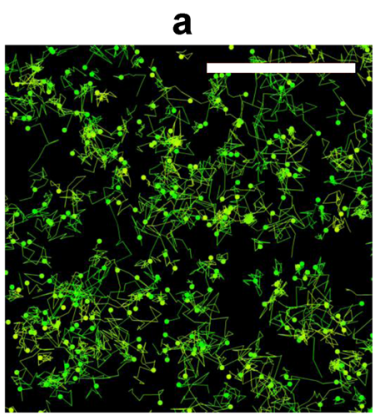

d

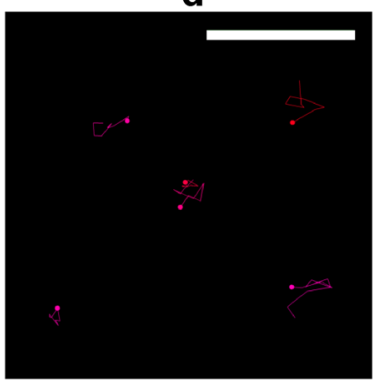

b

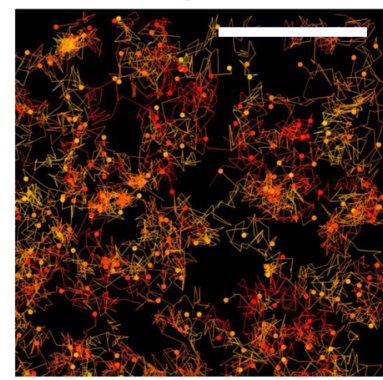

e

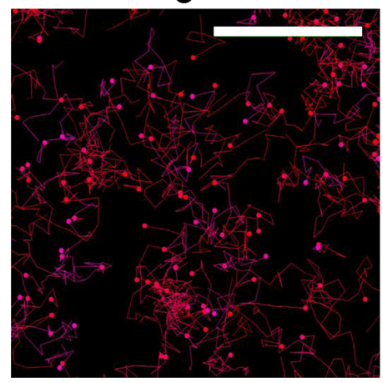

c

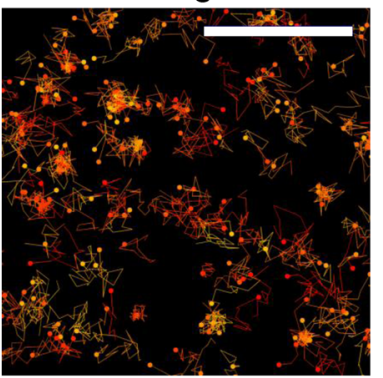

f

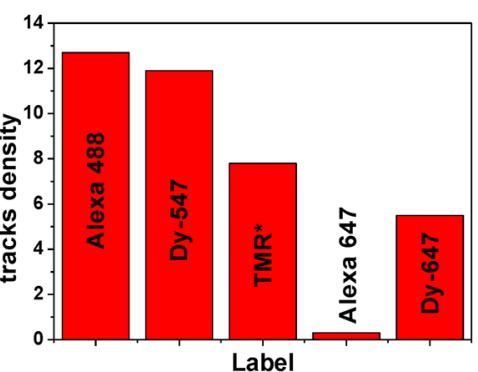

Figure 2. Tracks of $\beta 2 A R$ receptors fused to a SNAP-tag and labeled with (a) Alexa 488, (b) Dy-547, (c) TMR*, (d) Alexa 647, and (e) Dy-647. The starting point of each track is indicated by a dot. (f) Track density obtained for 5000 frames in Leibovitz media for these different dyes (minimum track size: 8 points). Scale bar $=2 \mu \mathrm{m}$.

blinking of several different dyes. ${ }^{14,15}$ Although live-cell imaging is possible for a limited time in such a buffer, ${ }^{8}$ we restricted our screen to combinations of less cytotoxic components previously used for super-resolution imaging, including the reducing agents glutathione e $^{16-18}$ and ascorbic acid. ${ }^{19,20}$ We tested buffer conditions using cells transiently transfected with the $\beta 2$ adrenergic receptor coupled to the SNAP-tag (SNAP- $\beta 2 A R$ ) and labeled with Alexa 488. Cells were exposed to moderate levels of excitation light to maintain cell viability while effectively putting most of the fluorophores in the dark state, and a stack of images was collected. These images were analyzed to localize single molecules by Gaussian fitting. At the same time, we could extract their photon counts in each frame, a measure of their brightness (Figure 1b). The molecular positions were then subjected to an automated tracking algorithm $^{1,21}$ to reconnect molecular trajectories as a function of time (Figure 1a): molecular localizations were connected as belonging to one molecular trajectory if they were present in consecutive frames and within a distance calculated based on the expected diffusion coefficient of the protein. To ensure correct trajectory reconnection, the density of fluorescent molecules per frame was kept low. Track densities, as defined by the number of tracks per area lasting more than eight frames, were compared for different buffers (Figure 1c). We found that by both quantitative measures (brightness and track density), a combination of Leibovitz medium, glutathione, and a lowconcentration enzymatic oxygen scavenger system composed of glucose oxidase and catalase was optimal for Alexa 488. Under these conditions, on average more than 400 photons were detected per localization, and more than 16 trajectories per square micrometer were reconstructed over a series of 5000 image frames. Surprisingly, Leibovitz medium alone yields a high number of tracks for Alexa 488 without requiring a complex mixture of buffers (Figure 1c); this is a promising result since Leibovitz is frequently used as a medium for imaging live cells in culture over a span of hours in the absence 
of $\mathrm{CO}_{2}$. Therefore, we subsequently used the Leibovitz buffer as a starting point for testing other dyes for multicolor tracking applications.

The primary obstacle to making several fluorophores blink simultaneously is in identifying a buffer that is appropriate for all the dyes used. In super-resolution imaging, this balance is necessary to achieve both high localization accuracy and a high density of molecules, as required by the Nyquist criteria. ${ }^{22}$ In the case of tracking, live imaging further restricts the possible buffer conditions. On the other hand, the Nyquist criteria no longer applies in single particle tracking, since the goal is rather to obtain a statistically significant number of molecular trajectories and does not require complete sample coverage. As a consequence, it is acceptable to use a buffer that yields a lower density of molecules than required for super-resolution imaging. Conversely, the constraints on the on-times of dyes are more stringent in single molecule tracking, as one needs sufficiently long on-times relative to the acquisition rate to obtain long trajectories of single molecules. Optimum conditions are therefore different for imaging and tracking.

We tested buffers and dyes for multicolor live-cell tracking using cells transfected with SNAP- $\beta 2 A R$ and labeled with either Alexa 488, TMR*, Dy-547, Alexa 647, or Dy-647. In screening a range of buffers, we found that Leibovitz medium allowed us to track dyes with emission in the green, red, and far-red parts of the visible spectrum (Figure 2). Interestingly, Alexa 647, which is one of the best dyes for super-resolution imaging, ${ }^{15}$ turns out to be a poor dye for tracking performed in Leibovitz. This underscores the difficulty in predicting which dyes will perform well in a live-cell setting based on in vitro or fixed cell data.

To enable multicolor single molecule tracking, it is necessary to target dyes with well-separated spectra to different proteins using orthogonal labeling schemes. Thus, we tested the SNAP-, Halo-, and ACP-tags fused, respectively, to $\beta 2 \mathrm{AR}$, transferrin receptors (TfR), or a glycosylphosphatidylinositol anchor sequence (GPI). In Table 1, we list the dyes that were used

Table 1. Summary of the Dyes Tested for Membrane Tracking in Leibovitz Medium

\begin{tabular}{ccccc} 
name & color & TAG & tracks density & permeability \\
Alexa 488 & green & Halo/SNAP & ++ & no \\
Dy-488 & green & ACP & + & no \\
Oregon Green & green & Halo/SNAP & + & yes \\
TMR* & red & Halo/SNAP & + & yes \\
Dy-547 & red & SNAP & ++ & no \\
Alexa 647 & far-red & Halo/SNAP & - & no \\
Dy-647 & far-red & ACP/SNAP & + & no \\
\hline
\end{tabular}

with each tag as well as rating their performance and cell permeability. There was no detectable difference when the same dye was targeted to a different tag on the same protein, indicating that the tag itself does not play an important role in dye performance. We note that the combination of Alexa 488 (Halo), TMR* (SNAP), and Dy-647 (ACP) represents three orthogonal tags that were fused to spectrally separated dyes, which all performed well in Leibovitz buffer alone (Figure 1, Supporting Information).

Once compatible blinking buffers and orthogonal labeling schemes have been established, it becomes possible to perform multicolor tracking. We cotransfected cells with the plasmamembrane receptor proteins SNAP- $\beta 2 \mathrm{AR}$ and Halo-TfR. Cells were colabeled with Dy-547 (SNAP) and Alexa 488 (Halo) and imaged sequentially. Overlaid maps of trajectories from each species reveal distinct zones of occupation for each protein (Figure 3c), suggesting spatial decorrelation. A closer analysis demonstrates that the distribution of mobilities as parametrized by the diffusion coefficient is similar for each receptor, with values in agreement with those measured using other methods. ${ }^{23,24}$ Also similar to single molecule tracking with other methods, we observed distinct classes of trajectories: an immobile fraction (Figure 3e, left), confined motion (Figure 3f, left), and directed motion (Figure $3 \mathrm{e}, \mathrm{f}$, right).

Imaging buffer conditions are crucial for controlling dye blinking. Traditionally used buffers usually contain BME or MEA which are cytotoxic, so it is challenging but important to find conditions which would be good in terms of both dye blinking and cell viability for live-cell imaging and tracking. We tested the different imaging buffers used in this work to quantify their cytotoxicity by performing the MTT survival assay. ${ }^{25}$ Cells were exposed to a buffer for approximately $2-3 \mathrm{~h}$, and the MTT test was run $24 \mathrm{~h}$ later. Our results indicate that cells incubated in all imaging buffers remain viable at least for $24 \mathrm{~h}$ after incubation, and the number of cells in the system was not significantly decreased compared to control experiments (Figure 2, Supporting Information). From this, we conclude that the buffer conditions used here are live-cell compatible.

An even greater challenge arises in performing single particle tracking on proteins located intracellularly. Because the local environment in a living cell is determined largely by cellular homeostasis, it is difficult and probably not desirable to strongly perturb these conditions. Thus, creating the appropriate environment for dyes to blink depends heavily on naturally occurring cellular processes. Moreover, relatively few dyes are cell permeable, further limiting the possibilities for tracking on intracellular targets. Of the handful of commercially available cell-permeable dyes that can be targeted using the SNAP-, CLIP-, or Halo-tags, we focused on three: Oregon Green, Dy505, and TMR*.

Mitochondria are enclosed by two membranes, with a proton gradient across the inner membrane that drives the synthesis of cellular ATP via the citric acid cycle. They represent a unique chemical environment, which could impact the blinking of dyes within the compartment. It was recently demonstrated that the spontaneous blinking of TMR* in mitochondria allows tracking of Halo-tagged proteins in the inner or outer mitochondrial membrane. ${ }^{9}$ We transfected cells with the inner mitochondrial membrane protein Cox8A-SNAP and found that Cox8ASNAP-TMR* labeled mitochondria specifically (Figure 4a), and that under optimized imaging conditions (Supporting Information), it blinked well and yielded a high density of molecules that could be rendered as a super-resolution image (Figure 4b). A large number of tracks were reconstructed from the molecular localizations and allowed us to create a spatial map of the determined mean diffusion coefficient. This map reflects the limited spatial variations of the diffusion coefficient, except at the edge of mitochondria where the reduced diffusion could be due to the fact that we measure $2 \mathrm{D}$ diffusion in a $3 \mathrm{D}$ sample (Figure 4c,d).

The nucleus represents another cellular compartment with unique chemical characteristics. We tested the possibility of tracking dyes fused to proteins in the nucleus. Cells were transfected with the histone protein $\mathrm{H} 2 \mathrm{~B}-\mathrm{SNAP}$ and labeled with Oregon Green, Dy-505, or TMR* which were previously used to demonstrate live-cell super-resolution imaging of 


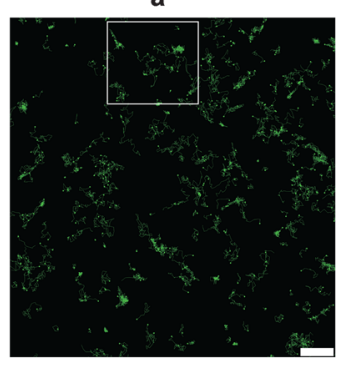

d

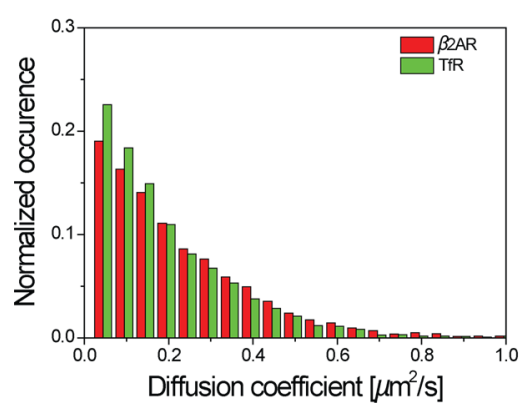

b
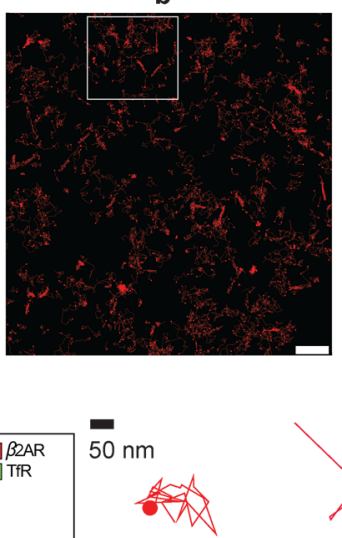

$\mathrm{D}=0.006 \mu \mathrm{m}^{2} / \mathrm{s}$

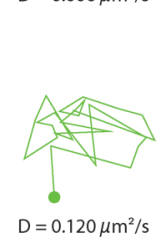

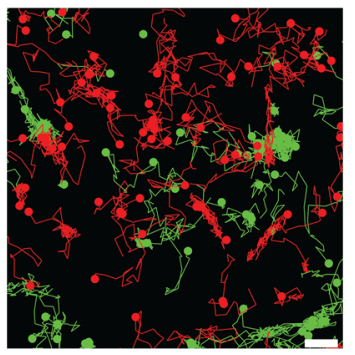

e

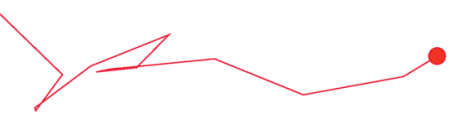

$\mathrm{D}=0.682 \mu \mathrm{m}^{2} / \mathrm{s}$

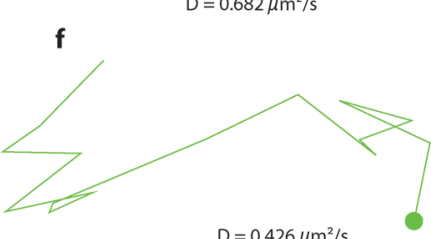

Figure 3. Tracks of (a) TfR-Halo-Alexa 488 and (b) SNAP- $\beta 2 A R-D y-547$ (scale bars: $=2 \mu \mathrm{m}$ ). The starting point of each track is indicated by a dot. (c) Zoom of the overlaid tracks in the region of interest delimited in (a) and (b) (scale bar $=500 \mathrm{~nm}$ ). (d) Distribution of the diffusion coefficient of the mobile fractions $\left(D>0.02 \mu \mathrm{m}^{2} / \mathrm{s}\right.$ ) of the two receptors (TFR:4770 tracks, $\beta 2 \mathrm{AR}: 2120$ tracks). Example of individual tracks of $\beta 2 \mathrm{AR}$ (e) and $\operatorname{TfR}(\mathrm{f})$ and associated diffusion coefficient.
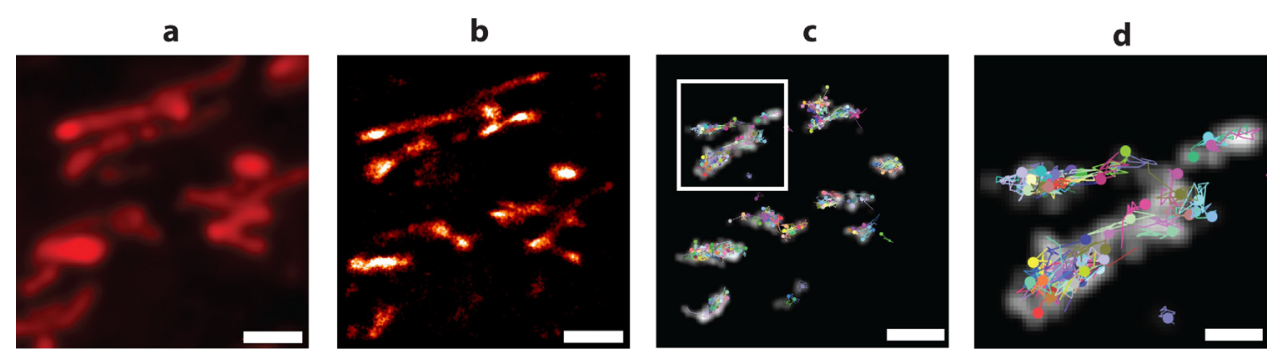

Figure 4. (a) Wide-field image of Cox8A-SNAP stained with TMR*. (b) Reconstructed super-resolution image. (c) Overlay of tracks from reconnected single molecule localizations and the measured map of diffusion coefficients color coded in gray (see Supporting Information). Higher intensities correspond to higher diffusion coefficients. The starting point of each track is indicated by a dot (scale bars $=2 \mu \mathrm{m})$. (d) Zoom of the region of interest delimited in $(c)($ scale bar $=700 \mathrm{~nm})$.

H2B. ${ }^{7,26}$ We found that all three dyes could be made to blink, and we used them both to create live-cell super-resolution images (Figure 5a) and track the position of $\mathrm{H} 2 \mathrm{~B}$ proteins (Figure $5 b, c$ ). We found the protein to be essentially immobile within the nucleus, confirming predictions based on fluorescence recovery after photobleaching experiments, which showed no recovery over a time scale of hours. ${ }^{27}$ Although all three dyes could be tracked at high density, the number of molecules localized over time was the most stable for TMR*, indicating its superior performance. Intriguingly, when these same dyes were targeted to cytoskeletal proteins, they did not undergo the sustained blinking necessary for high-density tracking in the imaging conditions used here. Such a striking difference in dye properties between the cytosol and the nucleus can be attributed at least in part to the differences in their chemical environments. Cellular compartments may contain different levels of natural reducing agents as well as molecular oxygen. This can have a strong impact on dye photoswitching behavior and could explain the observed differences in blinking.
Here we have demonstrated that synthetic dyes in different regions of the spectrum can be made to blink in a single buffer, chosen for its compatibility with single molecule tracking and long-term imaging in live cells. Targeted to specific proteins with orthogonal tags, these dyes can then be combined for multicolor imaging. With the dyes, tags, and buffers described here, up to three different species of proteins can be tracked on the surface of a single cell. Intracellular imaging is also possible with this method, as we show with mitochondria and the nucleus, illustrating the flexibility of this approach to highdensity molecular tracking. We also demonstrated the possibility of two-color single particle tracking in the nucleus. We expect that an increasing number of dyes will be designed and synthesized to be cell permeable and compatible with livecell imaging, further extending the range of possible protein targets. The future of this method is to combine the best features of existing techniques in high-density single molecule tracking, to allow the maximum flexibility in choice of proteins and colors. 

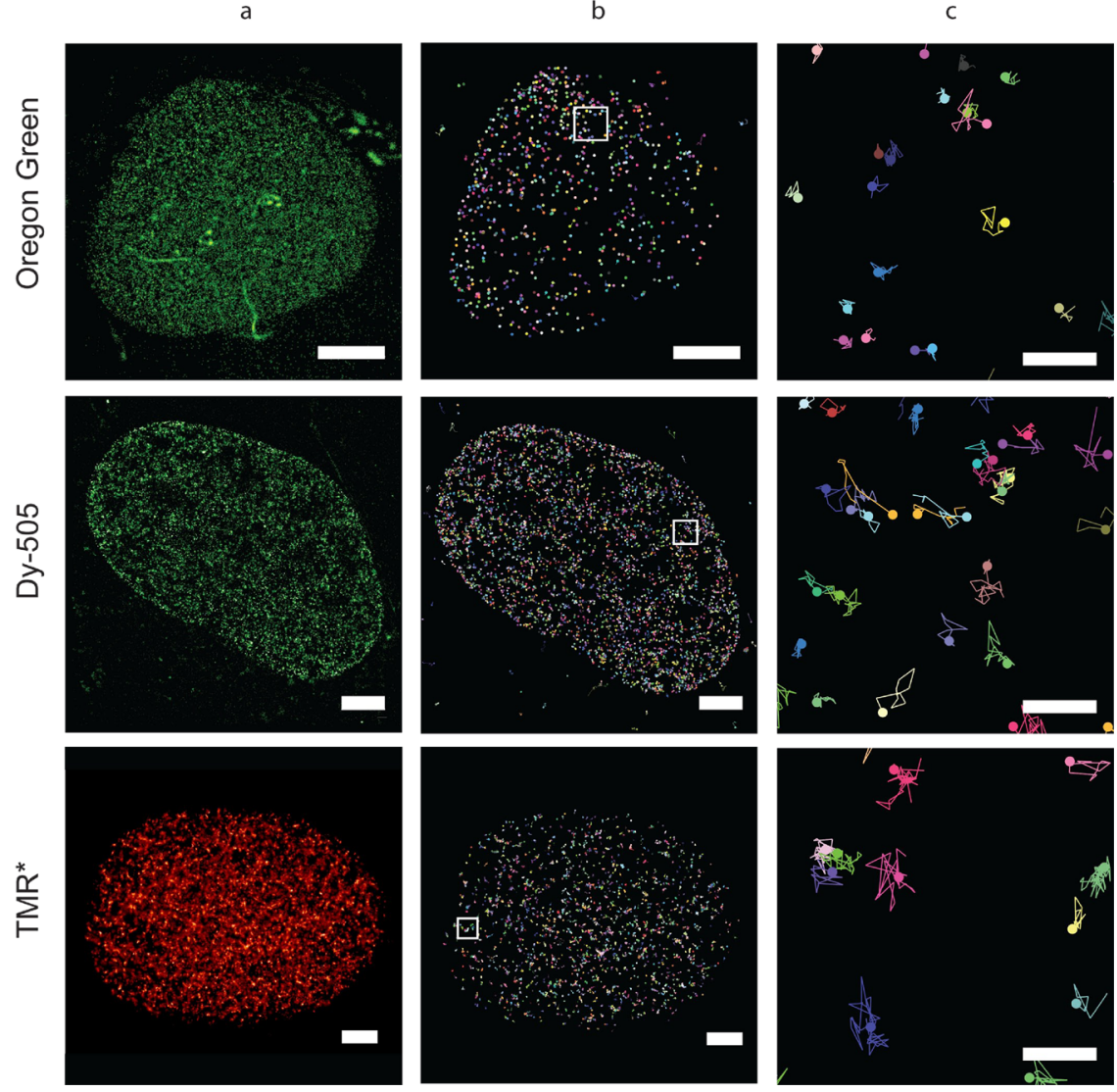

Figure 5. (a) Reconstructed super-resolution image of H2B-SNAP stained with different dyes (scale bars $=2 \mu \mathrm{m}$ ). Overlay of tracks from reconnected single molecule localizations (b) for the whole nucleus (scale bars $=2 \mu \mathrm{m}$ ) and (c) for zoom of the boxed region $(\mathrm{scale}$ bars $=100 \mathrm{~nm}$ ). The starting point of each track is indicated by a dot. Different rows correspond to cells labeled with Oregon Green, Dy-505, and TMR* (from top to bottom).

\section{ASSOCIATED CONTENT}

\section{S Supporting Information}

This material is available free of charge via the Internet at http://pubs.acs.org.

\section{AUTHOR INFORMATION}

\section{Corresponding Author}

*E-mail: suliana.manley@epfl.ch.

\section{Author Contributions}

₹These authors contributed equally.

\section{Notes}

The authors declare no competing financial interest.

\section{ACKNOWLEDGMENTS}

We thank Kai Johnsson for the gift of SNAP- $\beta 2 A R$ constructs and for BG- and Halo-dyes, Horst Vogel for the GPI-ACP construct, and Harald Hess for the use of Peakselector software. The research leading to these results has received funding from the European Research Council under the European Community's Seventh Framework Programme/ERC grant agreement number 243016-PALMassembly. The NCCR Chemical Biology, funded by the Swiss National Science Foundation, also supported this research.

\section{REFERENCES}

(1) Manley, S.; Gillette, J. M.; Patterson, G. H.; Shroff, H.; Hess, H. F.; Betzig, E.; Lippincott-Schwartz, J. Nat. Methods 2008, 5, 155-157.
(2) Giannone, G.; Hosy, E.; Levet, F.; Constals, A.; Schulze, K.; Sobolevsky, A.; Rosconi, M. P.; Gouaux, E.; Tampé, R.; Choquet, D.; Cognet, L. Biophys. J. 2010, 99, 1303-1310.

(3) Hinner, M. J.; Johnsson, K. Curr. Opin. Biotechnol. 2010, 21, 766-776.

(4) Keppler, A.; Gendreizig, S.; Gronemeyer, T.; Pick, H.; Vogel, H.; Johnsson, K. Nat. Biotechnol. 2005, 21, 86-89.

(5) Los, G. V.; et al. ACS Chem. Biol. 2008, 3, 373-382.

(6) Lee, H.-1. D.; Lord, S. J.; Iwanaga, S.; Zhan, K.; Xie, H.; Williams, J. C.; Wang, H.; Bowman, G. R.; Goley, E. D.; Shapiro, L.; Twieg, R. J.; Rao, J.; Moerner, W. E. J. Am. Chem. Soc. 2010, 132, 15099-15101.

(7) Klein, T.; Löschberger, A.; Proppert, S.; Wolter, S.; Van De Linde, S.; Sauer, M. Nat. Methods 2011, 8, 7-9.

(8) Jones, S.; Shim, S.-H.; He, J.; Zhuang, X. Nat. Methods 2011, 8, 499-505.

(9) Appelhans, T.; Richter, C. P.; Wilkens, V.; Hess, S. T.; Piehler, J.; Busch, K. B. Nano Lett. 2012, 12, 610-616.

(10) Betzig, E.; Patterson, G. H.; Sougrat, R.; Lindwasser, O. W.; Olenych, S.; Bonifacino, J. S.; Davidson, M. W.; Lippincott-Schwartz, J.; Hess, H. F. Science 2006, 313, 1642-1645.

(11) Rust, M. J.; Bates, M.; Zhuang, X. Nat. Methods 2006, 3, 793796.

(12) Hess, S. T.; Girirajan, T. P.; Mason, M. D. Biophysical Journal 2006, 91, 4258-4272.

(13) Heilemann, M.; van de Linde, S.; Schüttpelz, M.; Kasper, R.; Seefeldt, B.; Mukherjee, A.; Tinnefeld, P.; Sauer, M. Angew. Chem., Int. Ed. 2008, 47, 6172-6176.

(14) Van De Linde, S.; Löschberger, A.; Klein, T.; Heidbreder, M.; Wolter, S.; Heilemann, M.; Sauer, M. Nat. Protoc. 2011, 6, 991-1009.

(15) Dempsey, G.; Vaughan, J.; Chen, K.; Bates, M.; Zhuang, X. Nat. Methods 2011, 8, 1027-1040. 
(16) van de Linde, S.; Kasper, R.; Heilemann, M.; Sauer, M. Appl. Phys. B: Lasers Opt. 2008, 93, 725-731.

(17) Rasnik, I.; McKinney, S. A.; Ha, T. Nat. Methods 2006, 3, 891893.

(18) Cordes, T.; Vogelsang, J.; Tinnefeld, P. J. Am. Chem. Soc. 2009, 131, 5018-5019.

(19) Steinhauer, C.; Forthmann, C.; Vogelsang, J.; Tinnefeld, P. J. Am. Chem. Soc. 2008, 130, 16840-16841.

(20) Benke, A.; Manley, S. ChemBioChem 2012, 13, 298-301.

(21) Crocker, J. C.; Grier, D. G. J. Colloid Interface Sci. 1996, 179, 298-310.

(22) Shroff, H.; Galbraith, C. G.; Galbraith, J. A.; Betzig, E. Nat. Methods 2008, 5, 417-423.

(23) Kaya, A.; Ugur; Altuntas, O.; Sayar, K.; Onaran, H. O. Biochim. Biophys. Acta, Mol. Cell Res. 2011, 1813, 1511-1524.

(24) Hegener, O.; Prenner, L.; Runkel, F.; Baader, S. L.; Kappler, J.; Häberlein, H. Biochemistry 2004, 43, 6190-6199.

(25) Mosmann, T. J. Immunol. Methods 1983, 65, 55-63.

(26) Wombacher, R.; Heidbreder, M.; van de Linde, S.; Sheetz, M. P.; Heilemann, M.; Cornish, V. W.; Sauer, M. Nat. Methods 2010, 7, 717.

(27) Kimura, H.; Cook, P. R. J. Cell Biol. 2001, 153, 1341-1353. 